\title{
Interaction of fault ride through requirements and loss of mains protection
}

\author{
O. Raipala ${ }^{1}$, A. S. Mäkinen ${ }^{1}$ S. Repo ${ }^{1}$ and P. Järventausta ${ }^{1}$ \\ Department of Electrical Energy Engineering \\ Tampere University of Technology \\ Korkeakoulunkatu 3, FI-33720 Tampere (Finland) \\ Phone: +358 3311 511, e-mail: Ontrei.raipala@tut.fi
}

\begin{abstract}
The strongly growing DG capacity is raising concerns related to unintentional islanding. Unintentional islanding is prohibited due to the associated safety risks, and it is therefore mandatory to equip all DG units with some kind of loss of mains (LOM) protection. However, large amounts of unnecessary tripping of DG units cannot be tolerated anymore as the share of DG has already reached a significant share of the total installed power generation in certain regions. Certain grid codes thus require DG units to be able to ride through remote faults and support the power system. It is generally known that grid codes and LOM protection objectives are of somewhat controversial. However, it appears that no papers have studied the relation of LOM protection performance and fault ride through requirements thoroughly with simulations. This paper aims to fill this gap by providing extensive simulations which show how exactly the grid code requirements affect the performance of LOM protection. The studies are performed in a unique simulation environment consisting of two different types of real time simulators and a real LOM relay.
\end{abstract}

\section{Key words}

Loss of mains protection, anti-islanding protection, FRT requirements, Non-detection zone, real time simulation

\section{Introduction}

The rapidly increasing amount of DG has raised concerns related to unintentional islanding. Unintentional islanding is prohibited due to the associated safety risks which are:

1) Unsynchronized reclosing which may damage network components and DG units

2) Failed reclosing of a distribution feeder due to DG back feeding

3) Customer devices may be damaged due to poor power quality in the islanded circuit

4) Lines that are thought to be de-energized can be energized by DG. This is a safety risk for utility field personnel.

All DG units thus need to be equipped with some type of loss of mains (LOM) protection which ensures that unintentional islanding does not occur. The operation speed requirement for LOM protection may vary from case to case. For instance, the utilization of fast reclosing, i.e. aforementioned reasons number 1 and 2, require rapid islanding detection times from LOM protection (typically from $0.2 \mathrm{~s}$ to a couple of seconds). These requirements can be avoided by not using reclosing. However, this is highly undesirable from supply reliability point of view since the majority of faults are temporary in nature and can thus be cleared with the help of reclosing. For instance, in Finland about $90 \%$ of faults on overhead lines are temporary in nature and thus also clearable by automatic reclosing [1]. A reasonable option is to extend the open time of the circuit breaker during fast reclosing to provide enough time for LOM protection to operate.

Very sensitive LOM protection settings, however, also have disadvantages. This stems from the fact that faults in transmission network can launch huge amounts of adverse tripping of DG units which was, for instance, seen during the UCTE disturbance in the $4^{\text {th }}$ of November 2006 [2]. Because of this risk, system operators have issued grid codes that define how long generating units have to be able to stay connected and support the system stability during various kinds of disturbances. These fault ride through (FRT) requirements were originally meant only for large wind parks connected to high voltage (HV) grids. However, the rapid growth of DG has led these requirements to diffuse to medium voltage (MV) and low voltage (LV) levels as well.

It is generally known that the objectives of LOM protection are of somewhat controversial with the objectives of FRT requirements. However, it appears that no papers have studied the relation of LOM protection performance and FRT requirements thoroughly with simulations. This paper aims to fill this gap by providing extensive simulations which show how exactly the grid code requirements affect the performance of LOM protection. The studies are performed in a unique simulation environment consisting of two different types of real time simulators and a real LOM relay.

\section{Non-detection zone of LOM protection}

The non-detection zone (NDZ) is a suitable approach for assessing the performance of different LOM protection 
algorithms. NDZs can be represented in a load parameter space [3], [4] or in a power mismatch $(\Delta \mathrm{P}, \Delta \mathrm{Q})$ space [5], [6]. Power mismatch space is suitable for the assessment of passive LOM detection methods, whereas, for the assessment of active LOM detection schemes, it is advisable to utilize load parameter space [3]. More information concerning the NDZ concept can be found from references [3] and [4].

The behaviour of voltage magnitude and frequency in an islanded circuit are largely dependent not only on the characteristics of the DG unit(s) in the island, but also on the characteristics of the islanded load(s). The loads used in islanding detection tests are usually modelled as parallel RLC circuits with a quality factor $\left(\mathrm{Q}_{\mathrm{f}}\right)$ ranging from 1.0 to 2.5. A quality factor value of 2.5 is typically utilized in North American standards even though it is higher than what would be expected for a typical parallel RLC load [7]. However, there have been plans to reduce the $Q_{f}$ of islanding test load from 2.5 to 1.0 [7]. The quality factor, which defines the relative energy storage and dissipation of an RLC circuit, is defined in IEEE-929-2000 standard for a parallel RLC circuit in equation 1 [3], [7], [8].

$$
Q_{f}=R \sqrt{\frac{L}{C}}
$$

\section{Simulation environment}

The simulation studies presented here were conducted using a unique real time environment consisting of two types of real time simulators. The dSPACE is a well proven tool for modelling control systems and power electronics, whereas, the RTDS provides very accurate real time electromagnetic transient simulation for power systems. This environment, which is depicted in Fig. 1, also enables the connection of real external devices to be connected to function as a part of the simulation. More information on the environment can be found from [9].

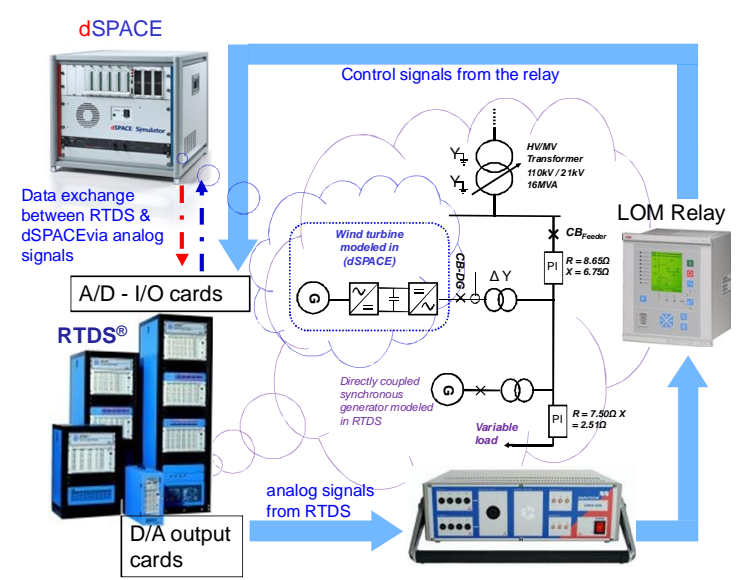

Fig. 1. The simulation environment

The RTDS, which consists of a number of processor cards and I/O cards, was used for running the power system modelled with the help of a RSCAD. A real LOM relay was set to control the DG unit circuit breaker in the power system model. The voltage signals from the connection point of the DG were first given as analogue output signals to a Omicron CMS156 amplifier, which amplified the signals to proper scale for the LOM relay. The LOM relay then sent its control decisions concerning the DG unit circuit breaker back to the RTDS as digital signals.

\section{A. Typical LOM relay settings}

The utilized LOM protection functions in the studies of this paper were undervoltage-, overvoltage, underfrequency and overfrequency protection. The LOM protection settings that were utilized for simulating the NDZs in Figs. 4 - 6, which are shown in table I, were not taken from any specific standard but they are very close to many European national recommendations [10].

Table I. - LOM protection settings

\begin{tabular}{|c|c|c|}
\hline Protection function & Threshold & delay \\
\hline Voltage & $0.8 \times \mathrm{Un} \& 1.15 \mathrm{x} \mathrm{Un}$ & $0.2 \mathrm{~s}$ \\
\hline Frequency & $49 \mathrm{~Hz} \& 51 \mathrm{~Hz}$ & $0.2 \mathrm{~s}$ \\
\hline
\end{tabular}

\section{B. FRT compliant LOM relay settings}

Low voltage ride through (LVRT) requirement is mostly related to loosening the undervoltage protection (UVP) threshold of LOM protection. However, certain other methods, as for instance rate of change of voltage, which are based on detecting islanding with the help of change in voltage, may also need to be loosened to allow the LVRT. The blue line in Fig. 2 illustrates the shape of LVRT curve for generating units in the range of $0.5 \mathrm{MW}$ to $100 \mathrm{MW}$ required by the Finnish transmission system operator Fingrid. Generating units need to be able to ride through faults in which the voltage does not drop below the blue curve in the figure, which represents the HV connection point voltage in per unit scale. The red line in Fig. 2 represents the two step approximation of the FRT curve which was utilized in the LOM relay.

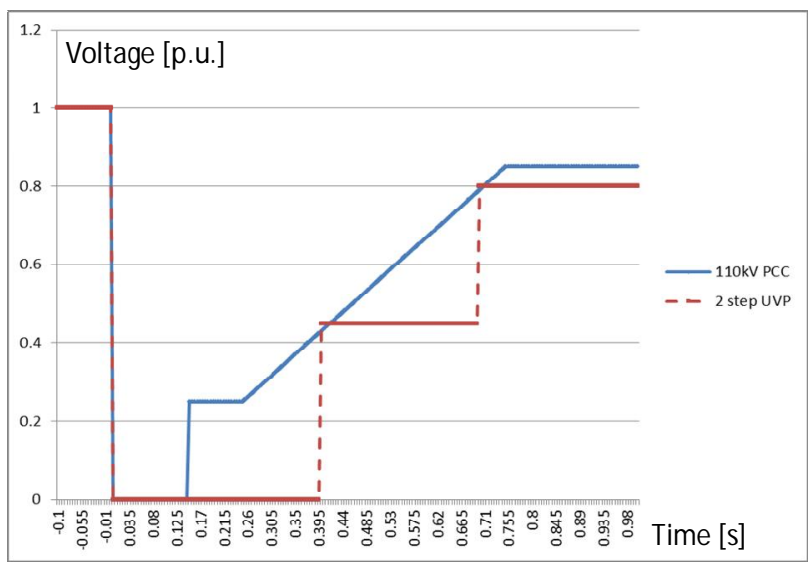

Fig. 2. The FRT requirement curve of Fingrid (blue line) and the utilized FRT compatible LOM protection UVP settings (red line)

The UVP settings had to be very simplified as shown in the figure because of the limited amount of configurable steps in the utilized protection relay. Relay manufacturers should take the FRT requirements into account in the UVP function blocks and design a user friendly way for making the UVP settings compatible with utility grid 
codes. The FRT compatible UVP settings are also shown in table II. However, modern grid codes require generating units not only to be able to ride through faults but also to be able to support the voltages by feeding reactive power during grid faults [11], [12]. This issue will be taken into account in the simulation studies presented in chapter 5 .

Table II. - The FRT compatible LOM protection settings

\begin{tabular}{|c|c|c|}
\hline Protection function & Threshold & delay \\
\hline Undervoltage low stage & $0.8 \times \mathrm{Un}$ & $0.7 \mathrm{~s}$ \\
\hline Undervoltage high stage & $0.45 \times \mathrm{Un}$ & $0.4 \mathrm{~s}$ \\
\hline
\end{tabular}

It is noteworthy that the FRT compatibility is also related to communication based LOM protection methods since remote methods are typically equipped with a local LOM protection method for back up purposes. Thus, without coordination, the local back up protection may cause unwanted tripping during voltage dips. On the other hand, applying such back up protection settings that will enable the FRT will naturally degrade the performance of back up protection. One option to avoid this problem is to use continuous supervision of the communication channel instead of back up protection and immediately disconnect the protected DG unit whenever a malfunction in the communication channel is detected. However, as this kind of approach also causes unwanted tripping of DG, it would be more reasonable to only switch to the use of local back up protection once a malfunction in the communication channel is detected. This would ensure reliable and FRT compatible LOM protection.

\section{Simulation models}

A simple distribution network model, which is shown in Fig. 3, was modelled with the help of RSCAD for performing these studies. The model consists of voltage source representing the main grid, a $110 \mathrm{kV} / 21 \mathrm{kV}$ rated $\mathrm{HV} / \mathrm{MV}$ transformer, one MV distribution feeder which is represented by two $\pi$-line representations and a variable load at the tail part of the feeder. All the above described components were simulated by the RTDS, whereas, the modelled full converter connected wind turbine unit was simulated by the dSPACE as shown in Fig. 3. The modelling of this 500kVA rated DG unit is based on [13]. The synchronization to the grid voltage is carried out using synchronous reference frame phase locked loop (SRFPLL) [14]. However, in one of the simulated cases another synchronization method was used in order to examine the significance of utilized synchronization method from LOM protection point of view. In this examined method, synchronization is implemented exploiting a PLL together with zero crossing detection of the phase a-supply voltage [15]. This method responds to changes fairly slowly since the zero crossing instants can only be detected once per half cycle of the utility voltage [15].

The power imbalance on the feeder was varied in small steps by varying the demand of the parallel RLC load connected to the tail part of the feeder. For each combination of power imbalance, the $\mathrm{CB}_{\text {Feeder }}$ switch was opened after the output power of the DG unit had stabilized. The resulting power island was then maintained only by the DG until the LOM relay operated. In each case, the active and reactive power imbalance, the operation time of the LOM relay as well as certain other parameters were captured. The NDZs for the studied cases, which will be presented in the following chapter, were determined based on this stored data.

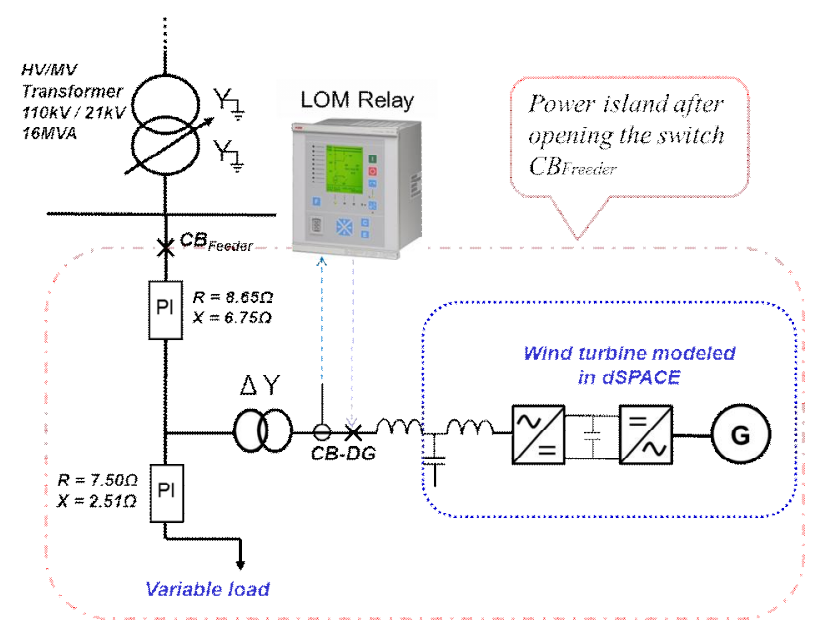

Fig. 3. The utilized simulation model

\section{Simulation studies}

This chapter, which presents the simulated NDZs, is divided into four subchapters. The first subchapter illustrates the effect of utilized synchronization method and the quality factor of the load on the performance of LOM protection. The second subchapter studies how exactly the performance of LOM protection is degraded when UVP function is set to allow the LVRT. The third subchapter demonstrates how reactive power support of DG affects the performance of LOM protection. Finally, the fourth subchapter examines how the addition of ROCOF function can enhance the situation. The quality factor was kept at 1.0 in all the simulation studies presented here except for the NDZ presented in Fig 6.

\section{A. NDZ of a typical LOM protection}

The LOM relay was configured according to the settings shown in table I in the studies of this subchapter. In the first simulated NDZ, the utilized synchronization method of the grid side converter (GSC) was a simple zero crossing based PLL (see [15] for more details). Fig. 4 shows the resulting NDZ. The quality factor of the load was kept at 1.0 in the simulations.

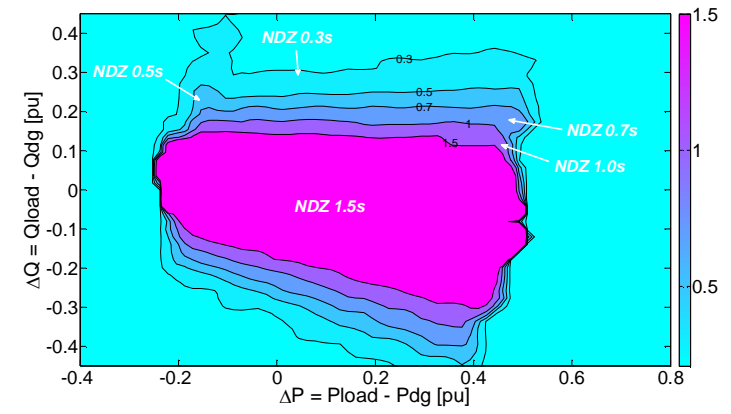

Fig. 4. The NDZ of LOM protection when the utilized synchronization method of the GSC was a PLL based on zero crossings. $\mathrm{Q}_{\mathrm{f}}$ was 1.0. 
The pink coloured area marked with the sign "NDZ 1.5s" in Figs. 4 - 9 represents the set of power imbalance combinations where LOM protection failed to isolate the DG unit within $1.5 \mathrm{~s}$ from the beginning of the islanding. The other layers marked with the signs "NDZ 1.0s", "NDZ $0.7 \mathrm{~s}$ ", "NDZ $0.5 \mathrm{~s}$ " and "NDZ $0.3 \mathrm{~s}$ " respectively refer to relay operation times $1.0 \mathrm{~s}, 0.7 \mathrm{~s}, 0.5 \mathrm{~s}$ and $0.3 \mathrm{~s}$. This means that the smaller the size of NDZ is, the better the performance of LOM protection is. This multi-layer NDZ format is more suitable for showing the effect of the FRT compatible LOM protection settings compared to the basic NDZ format as it will be seen from the later results.

In all the rest of the following simulations, the utilized synchronization method of the GSC was the SRF-PLL [14]. The resulting NDZ, which is shown in Fig. 5, is considerably smaller than the one in Fig 4. The comparison between Figs. 4 and 5 thus clearly illustrates that the importance of the utilized synchronization method of the grid side converter should not be underestimated.

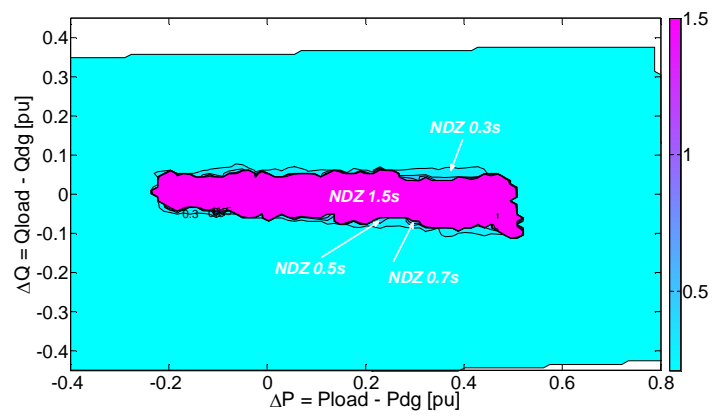

Fig. 5. The NDZ of LOM protection when the utilized synchronization method of the GSC was the SRF-PLL.

The quality factor of the parallel RLC load was now changed from 1.0 to 0.1 and the previous simulations were repeated. Fig. 6 shows the resulting NDZ. By comparing Figs. 5 and 6 it can be clearly seen that the size of the NDZ reduces significantly in the $\Delta \mathrm{Q}$ range as the quality factor of the parallel RLC load is reduced to 0.1 .

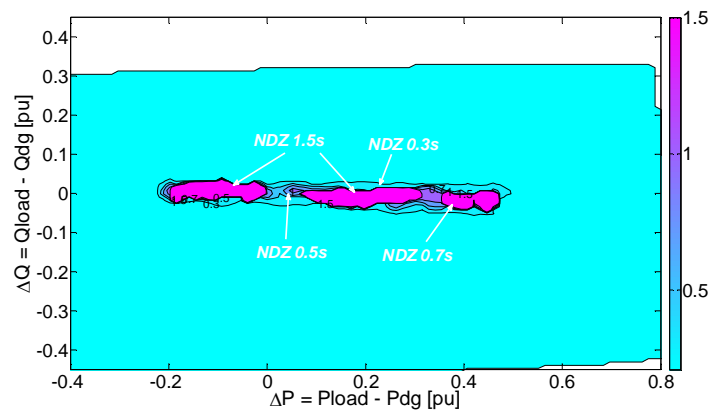

Fig. 6. The effect of reducing $\mathrm{Q}_{\mathrm{f}}$ to 0.1 .

\section{B. NDZ of FRT compliant LOM protection}

In the following case, the UVP function of the LOM relay was set to allow the LVRT by utilizing the settings presented in table II. Fig. 7 represents the resulting NDZ. It can be seen from the figure that the NDZ now extends considerably further in the positive $\Delta \mathrm{P}$ axis direction than what it did with the original settings (Fig. 5). It can be seen from Fig. 7 that the size of the NDZ area where it took more than $1.5 \mathrm{~s}$ to detect islanding is approximately same as in Fig. 5. However, a long extended NDZ area where it took more than $0.7 \mathrm{~s}$ is now added to the original NDZ. It is noteworthy that UVP function trip region was not reached in the simulated NDZ, that is, the NDZ would extend even further towards the positive $\Delta \mathrm{P}$ axis direction than what Fig. 7 shows. Note that the scaling in Fig. 7 is very different from the scaling in earlier figures. The quality factor was kept at 1.0.

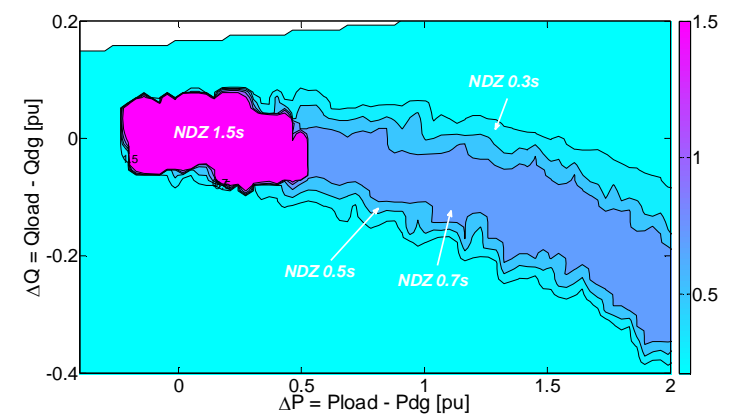

Fig. 7. NDZ of a FRT compatible LOM protection. $\mathrm{Q}_{\mathrm{f}}$ was 1.0.

A clear bending can be seen in Fig. 7. This is caused by a sequence of events. Firstly, let us analyze the power imbalances during islanding with the help of equations 2 and 3 . The relation between active power imbalance and voltage can be expressed with equation 2 , whereas, the relation between reactive power imbalance and the frequency can be expressed by equation 3 :

$$
\begin{gathered}
\Delta P=P_{\text {Load }}-P_{D G}=\frac{U^{2}}{R}-P_{D G} \\
\Delta Q=Q_{\text {Load }}-Q_{D G}=\frac{U^{2}}{X}-Q_{D G}=\frac{U^{2}}{2 \pi f L-\frac{1}{2 \pi f C}}-Q_{D G}
\end{gathered}
$$

where $\mathrm{U}$ refers to the voltage at the load node, $\mathrm{X}$ is the reactance of the load and $f$ refers to the frequency in the studied circuit. The losses are included in $\mathrm{P}_{\text {Load }}$ and $\mathrm{Q}_{\text {Load. }}$. Note that when an islanded circuit is sustained only by a converter connected DG unit, reactive power imbalance mainly determines the frequency and active power imbalance mainly determines the voltages in the island [4], [6]. Let us now consider a situation where a large initial active power deficiency is present in the circuit (i.e. a large $\Delta \mathrm{P}$ ) at the time when islanding occurs. It can be seen from equation 2 that due to the large $\Delta \mathrm{P}$ the voltage is forced to decrease in order to bring a new balance between production and consumption in the islanded circuit because $\mathrm{R}$ and $\mathrm{P}_{\mathrm{DG}}$ are fixed.

In this study, the GSC of the DG unit is controlled to operate with unity power factor during nominal operation mode. In order to achieve this goal, the reactive power generated by the LCL-filter capacitor of the GSC should be compensated. The compensation is done in the GSC control system by selecting a proper constant reactive current reference [9]. Thus, the GSC feeds a fixed constant inductive current component which compensates the reactive power produced by the filter capacitor at nominal voltage. However, when the voltage drops due to the large initial $\Delta \mathrm{P}$ value, the reactive power produced by the filter capacitor reduces proportionally to the square of the voltage, whereas, the compensation power drawn by the $\mathrm{DG}$ unit $\left(\mathrm{Q}_{\mathrm{DG}}\right.$ is only directly proportional to voltage. 
Hence, the DG unit consumes reactive power with large $\Delta \mathrm{P}$ values due to the reduced voltage. NDZ can only occur if there is a balance between produced and consumed reactive power. Hence, during significantly decreased voltage caused by large $\Delta \mathrm{P}$, the NDZ can exist only if the network load generates the reactive power $(\Delta \mathrm{Q}<0)$ which is consumed by the DG. As a consequence, the NDZ bends to the negative side of $\Delta \mathrm{Q}$.

\section{The effect of reactive power support of DG units}

As already mentioned, modern grid codes usually also require generating units to be capable of supporting the power system during voltage dips by feeding reactive power into the grid. In the following case, a voltage droop with a 5 percent deadband was added to the GSC control system. The droop was adjusted so that the DG unit gave maximum available reactive power output at 0.5 per unit voltage. Fig. 8 shows the resulting NDZ. As the figure illustrates, the NDZ area now covered surprisingly large reactive power imbalances. This result shows that the performance of LOM protection is dangerously degraded when DG units are required to both be able to ride through faults and to provide voltage support by feeding reactive power.

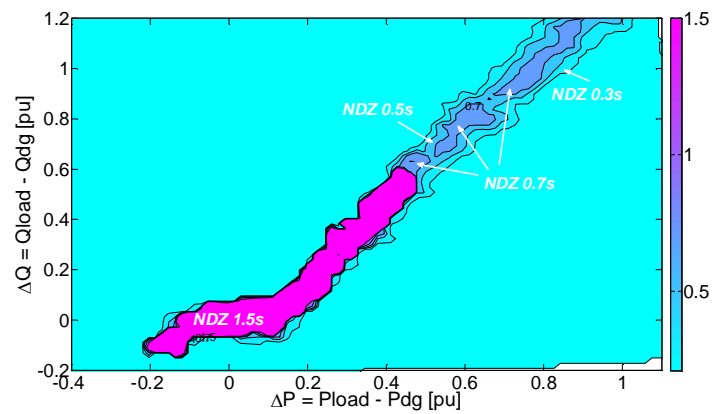

Fig. 8. The NDZ when a voltage droop control (5\% deadband) was added to the GSC control system. $\mathrm{Q}_{\mathrm{f}}$ was 1.0.

The reason for the bending of the NDZ in Fig. 8 is caused by the addition of the voltage droop. This can be understood by analysing the positive $\Delta \mathrm{P}$ half of the graph. The greater the value of $\Delta \mathrm{P}$ in this half of the figure, the greater is the decrease in the voltage at the DG- and at the load node. When the voltage drops below 95 percent of its nominal value at the DG connection point, the GSC begins to feed reactive power into the grid. Hence, an inductive load large enough has to be present in the islanded circuit in order for a NDZ to exist. The larger the initial $\Delta \mathrm{P}$ value, the greater is the reactive power production of the DG unit during islanding, and the larger is the required value of the inductive load for the NDZ to exist. This causes the NDZ to bend as shown in Fig. 8.

Similar reasoning can be used for understanding the bending in the negative $\Delta \mathrm{P}$ half of the Fig. 8 . The smaller the $\Delta \mathrm{P}$ value is, the more the voltage at the $\mathrm{DG}$ node increases. Thus, due to the voltage droop control, the DG unit begins to consume reactive power. Hence, a capacitive load that produces the reactive power consumed by the DG unit has to be present in order for the NDZ to exist. Consequently, the smaller the $\Delta \mathrm{P}$ value, the larger is the required value of the capacitive load for the NDZ to exist. This causes the NDZ to bend in the negative $\Delta \mathrm{P}$ half.

\section{The effect adding ROCOF function}

The rate of change of frequency (ROCOF) is one of the most utilized LOM protection methods. In the following case, the ROCOF function was added to the relay and its threshold was set to $1 \mathrm{~Hz} / \mathrm{s}$ with a $0.2 \mathrm{~s}$ operate delay time. Fig 9 shows the resulting NDZ. The comparison between Figs. 8 and 9 shows that the addition of ROCOF reduces the size of the NDZ considerably from its $\triangle \mathrm{Q}$ boundaries. However, the ROCOF is not able to reduce the size of the $\mathrm{NDZ}$ in the $\Delta \mathrm{P}$ direction as expected.

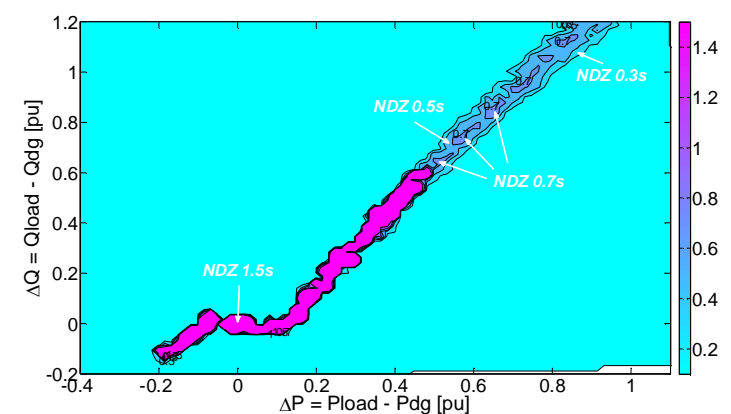

Fig. 9. The NDZ when the GSC was operated in voltage droop mode and the ROCOF function was set to $1 \mathrm{~Hz} / \mathrm{s}$. $\mathrm{Q}_{\mathrm{f}}$ was 1.0.

\section{Discussion}

Certain observations can be made from the simulated NDZs. Firstly, when analysing the effect of changing the normal UVP settings to FRT compatible settings, that is, comparing figures 5 and 7 , one can see that the performance of LOM protection degrades significantly when changing to LVRT compatible UVP settings. This degrading is seen from the NDZ area which extends further than 2 in per unit scale. However, it is not as easily seen whether the addition of voltage droop control makes the situation more difficult to LOM protection. This can be assessed by evaluating which power imbalance combinations in the NDZ area are actually probable. Loads are normally on the inductive side rather than capacitive side, i.e., loads consume some, although typically small amount of reactive power. On the other hand, DG units in MV and LV networks are at present usually operated at unity power factor, i.e., they neither consume nor produce reactive power. This means that in most cases there is a small deficiency of reactive power in islanded circuits prior to the islanding event. Thus, such power imbalance combinations where $\Delta \mathrm{Q}=\mathrm{Q}_{\text {load }}-$ $\mathrm{Q}_{\mathrm{DG}}>0$ are much more probable than those where $\Delta \mathrm{Q}<$ 0 as illustrated in Fig. 10.

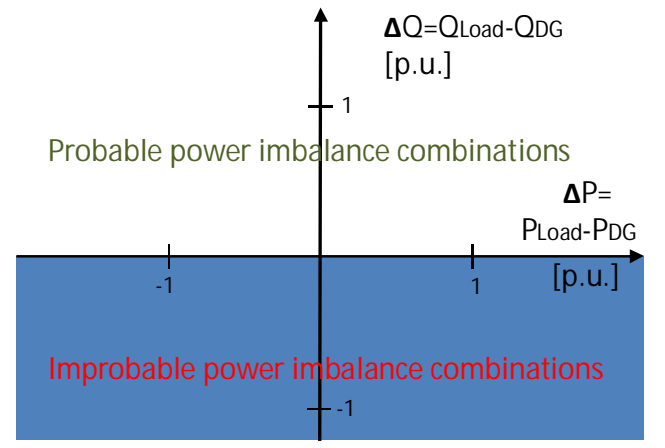

Fig 10. Probability of power imbalance combinations 
It is nevertheless possible that the power imbalance could be on the capacitive side if for instance some poorly dimensioned compensation equipment is installed in the islanded circuit. However, this is not common as network operators usually have additional fees for significant reactive power production and consumption in medium voltage network level for discouraging such behaviour.

When examining Fig. 7 with the help of the idea presented in Fig. 10, it can be observed that a large portion of the NDZ area is situated in the improbable $\Delta \mathrm{Q}$ range. This is illustrated in Fig. 11, which shows the improbable $\Delta \mathrm{Q}$ range in the NDZ which was already presented in Fig. 7. Now, by evaluating which part of the NDZ in Fig. 8 is situated in the improbable $\Delta \mathrm{Q}$ range, which is illustrated in Fig. 12, one can clearly observe that the situation becomes considerably more challenging to LOM protection when the voltage droop control is included.

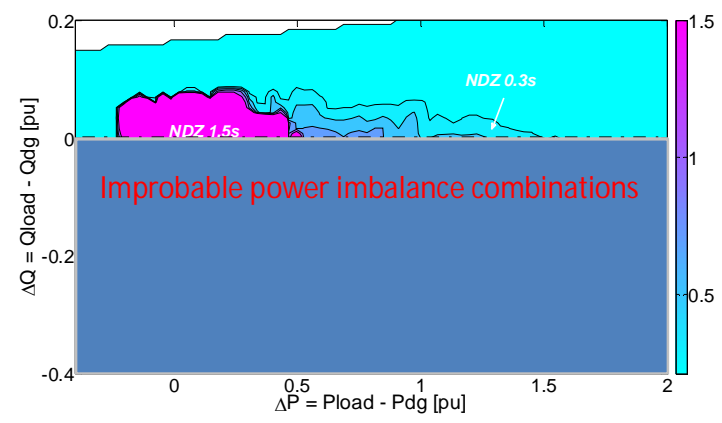

Fig. 11. The improbable power imbalance values in Fig. 7

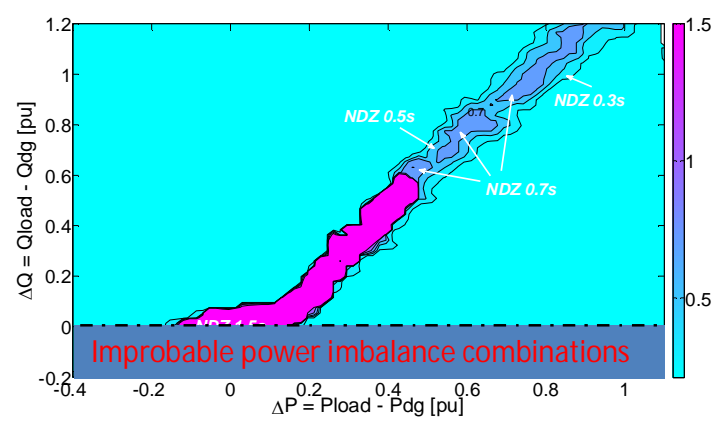

Fig. 12. The improbable power imbalance values in Fig. 8

However, in the future DG units may be utilized for controlling local voltages by consuming or producing reactive power. Voltage rise is probably a more common problem in the connection points of DG units than voltage drop. Thus, DG units would more likely be used for consuming reactive power and thus for preventing excessive voltage rise. This supports the idea presented in Fig. 10. However, DG units could in some cases also be used for producing reactive power and thus for preventing excessive voltage drop. This would lower the improbable power imbalance combinations boundary in Fig. 10.

\section{Conclusion}

This paper studied the effect of FRT requirements on LOM protection. The studies were based on a large number of simulations conducted in a unique real time simulation environment including a real LOM relay. It was observed that when LOM protection is set to allow DG units to ride through faults, the performance of UVP function is significantly degraded. In fact, in these studies, the NDZ region extended to further than 2 per unit in real power imbalance. Another observation was that the reactive power support which is required in many modern grid codes has a significant effect on the performance of LOM protection. Problematic situations for LOM protection may exist with surprisingly large reactive power imbalance points when the studied DG unit is set to support the system during voltage dips by feeding reactive power. It was also observed that the utilized synchronization method of the GSC may have a significant effect on the performance of LOM protection.

\section{References}

[1] Sähköenergialiitto ry \& Energia-alan Keskusliitto ry, Keskeytystilasto 2003, Helsinki (2004), 21p.

[2] UCTE, System Disturbance on 4 November 2006, Final Report

[3] L. A. C. Lopes and H. Sun, "Performance Assessment of Active Frequency Drifting Islanding Detection Methods", IEEE Trans. on Energy Conversion, Vol. 21, No. 1, pp. $171-180,2006$

[4] M. Ropp et al., "Determining the Relative Effectiveness of Islanding Detection Methods Using Phase Criteria and Nondetection Zones", IEEE Trans. on Energy Conversion, Vol. 15, No. 3, pp. 290 - 296, 2000

[5] J. C. M. Vieira, W. Freitas, W. Xu and A. Morelato, "An Investigation on the Nondetection Zones of Synchronous Distributed Generation Anti-Islanding Protection", IEEE Trans. on Power Delivery, Vol. 23, No. 2, 2008

[6] Z. Ye, A. Kolwalkar, Y. Zhang, P. Du and R. Walling, "Evaluation of Anti-Islanding Schemes Based on Nondetection Zone Concept", IEEE Trans. on Power Electronics, Vol. 19, No. 5, 2004

[7] W. Xu, K. Mauch and S. Martel, an Assessment of Distributed Generation Islanding Detection Methods and Issues for Canada, CETC-Varennes 2004-074 (TR) 411INVERT, Canada (2004), 53p.

[8] IEEE Std. 929-2000, IEEE Recommended Practice for Utility Interface of Photovoltaic (PV) Systems, Sponsored by IEEE Standards Coordinating Committee 21 on Photovoltaics, New York (2000), 26 p.

[9] A. Mäkinen and H. Tuusa, "Wind Turbine and Grid Interaction Studies Using Integrated Real-Time Simulation Environment", in Proc. NORPIE2008

[10] V. Van Thong and R. Belmans, "Maximum penetration level of distributed generation with safety criteria", European Trans. on Electric Power, Vol. 20, No. 3, pp. 367-381, 2010

[11] E. ON Netz GmbH, Requirements for Offshore Grid Connections in the E.ON Netz network, E. ON Netz GmbH, Bayreuth (2008)

[12] VDN, TransmissionCode 2007 Netz- und Systemregeln der Deutschen Übertragungsnetzbetreiber. Germany (2007), 90p.

[13] A. S. Mäkinen, H. Tuusa, "Impact of strength of fault current path on the operation of decoupled double synchronous reference frame - phase locked loop", to be published in ICREPQ'13 in March 2013

[14] R. Teodorescu, M. Liserre and P. Rodríguez, Grid Converters for Photovoltaic and Wind Power Systems, John Wiley \& Sons, Ltd., UK (2011), 398p.

[15] M. Routimo, Developing a Voltage-Source Shunt Active Power Filter for Improving Power Quality, Doctoral thesis, Tampere University of Technology, Tampere (2008), 86p. 